\title{
Exposição aguda ao Metilmercúrio em ratos em desenvolvimento: mini-revisão
}

\section{Acute exposition of developing rats to Methylmercury: a mini-review}

\author{
Paula E. R. Bitencourt ${ }^{1}$; Faida H. Abdalla ${ }^{1}$; Karine S. De Bona ${ }^{2}$; Maria B. Moretto ${ }^{3}$
}

\section{Resumo}

Mercúrio $(\mathrm{Hg})$ está presente no ambiente em três diferentes formas químicas: elementar $\left(\mathrm{Hg}^{0}\right)$, inorgânico e orgânico, sendo que a sua distribuição, toxicidade e metabolismo são dependentes de sua forma química. A exposição oral ao consumo de peixes e alimentos contaminados é a principal forma de exposição humana ao metilmercúrio $(\mathrm{MeHg})$, um poluente ambiental que é absorvido por ingestão, inalação e através da pele. $\mathrm{O} \mathrm{MeHg}$ é um potente neurotóxico, especialmente para o cérebro em desenvolvimento. Neste estudo, foram examinados os principais efeitos da exposição ao $\mathrm{MeHg}$ durante o desenvolvimento salientando os mecanismos bioquímicos envolvidos nestes processos. Também foram apresentados recentes resultados sobre o uso de extratos de plantas medicinais que atenuaram os efeitos adversos deste metal. Deste modo, estes dados reforçam a toxicidade do $\mathrm{MeHg}$ durante o desenvolvimento e sugerem possíveis caminhos para futuras intervenções terapêuticas.

Palavras-Chave: Metilmercúrio. Ratos. Desenvolvimento.

\begin{abstract}
Mercury $(\mathrm{Hg})$ is present in the environment in three different chemical forms: elemental, inorganic and organic Hg. The distribution, toxicity and metabolism of $\mathrm{Hg}$ are linked to its chemical form. The oral exposition following fish and food contaminated is the main route of contamination of humans to the methilmercury (MeHg), an environmental pollutant which is absorbed by ingestion, inhalation and through the skin. $\mathrm{MeHg}$ is a strong neurotoxic molecule, especially for the developing brain. In this study, the main effects of the $\mathrm{MeHg}$ exposition and the biochemical parameters involved in this process were examined. The results of the use of plant extracts which attenuate the adverse effects of this metal are also presented here. Therefore, these data reinforce the MeHg toxicity during the development and suggest alternative ways for future therapeutic approaches.
\end{abstract}

Keywords: Methylmercury. Rats. Development.

\footnotetext{
${ }^{1}$ Pós graduanda do Programa de Pós Graduação em Ciências Farmacêuticas, da Universidade Federal de Santa Maria, UFSM.

${ }^{2}$ Pós graduanda do Programa de Pós Graduação em Farmacologia, da Universidade Federal de Santa Maria, UFSM.

${ }^{3}$ Farmacêutica Bioquímica, Mestre e Doutora em Bioquímica, Docente do curso de Farmácia da Universidade Federal de Santa Maria, UFSM. E-mail: beatriz@smail.ufsm.br.
} 


\section{Introdução}

O mercúrio $(\mathrm{Hg})$ é um elemento natural existente em todo o ambiente. É principalmente liberado como elementar $\left(\mathrm{Hg}^{0}\right)$ na atmosfera a partir de fontes naturais e antropogênicas, sendo que, estudos demonstram que a atividade humana contribui com cerca de $50-70 \%$ do total de emissões para o ambiente (UNITED NATIONS ENVIRONMENT PROGRAMME, 2002). Após a exposição ao metal, o mesmo é absorvido pelo trato gastrointestinal e ao chegar à circulação sanguínea, o Hg é oxidado formando complexos solúveis, os quais se combinam com proteínas do plasma e com eritrócitos (GOLDMAN; SHANNON, 2001). Dessa forma, eles são distribuidos para os tecidos, depositandose principalmente nos rins, fígado, sangue, cerébro, ossos e pulmões, causando danos nesses sistemas, especialmente, no Sistema Nervoso Central (SNC) (AUCOTT; MCLINDEN; WINKA, 2003). O Hg é encontrado em três diferentes formas: elementar, compostos orgânicos e inorgânicos (GOLDMAN; SHANNON, 2001). O metilmercúrio $(\mathrm{MeHg})$ é considerado o composto orgânico de $\mathrm{Hg}$ mais tóxico (AMIN-ZAKI et al., 1974; CLARKSON, 2002).

Na forma orgânica, o íon mercúrio apresenta-se ligado covalentemente a um radical orgânico, sendo o metilmercúrio $\left(\mathrm{CH}_{3} \mathrm{Hg}^{+}\right)$e o dimetilmercúrio $\left(\left(\mathrm{CH}_{3}\right)_{2} \mathrm{Hg}\right)$ os mais comuns, ou ainda com ligantes orgânicos naturais (CANELA, 1995; HORVAT, 1996; JARDIM, 1988). Uma vez formado, o metilmercúrio entra na cadeia alimentar através da rápida difusão e forte ligação com as proteínas da biota aquática, atingindo sua concentração máxima em tecidos de peixes, principal fonte de contaminação de $\mathrm{MeHg}$ (MICARONI; BUENO; JARDIM, 2000). O acúmulo de $\mathrm{MeHg}$ no organismo humano representa riscos significativos para a saúde. Entre eles, anemia, déficit cognitivo, tremores, gengivite, hipertensão, irritabilidade, câncer, depressão, perda de memória, insuficiência renal crônica, infertilidade e doença de Alzheimer (GRESSENS et al., 2001; KAKITA et al. 2000; SANFELIU; SEBASTIAN; KIM, 2001).
Modelos experimentais de toxicidade ao $\mathrm{MeHg}$ desenvolvidos em roedores demonstram que, assim como em humanos, existe uma alta afinidade desse agente tóxico ao SNC (HAYKAL-COATS et al., 1998). Os principais mecanismos envolvidos na neurotoxicidade do $\mathrm{MeHg}$ são a geração de estresse oxidativo, alteração da homeostase de cálcio intracelular e interferência com o transporte de membranas e alta afinidade por grupamentos sulfidrilas (JUAREZ et al., 2002; SHANKER et al., 2002; TROMBETA; KROMIDAS, 1992). Assim, o objetivo deste estudo foi examinar os efeitos da exposição ao $\mathrm{MeHg}$ durante o período de desenvolvimento em ratos.

\section{Metilmercúrio}

$\mathrm{O} \mathrm{MeHg}$ é a forma orgânica de mercúrio mais conhecida, pois é a que predomina no meio ambiente, sendo formado através da ação de microorganismos, os quais, metilam o mercúrio elementar presente na água, solo ou ar. O consumo de peixes é uma das principais formas de exposição humana a esse contaminante (ASCHNER et al., 2007; CARVALHO et al., 2008; SAKAMOTO et al., 2013).

O primeiro relato de intoxicação humana foi relatado a cerca de 150 anos atrás quando ocorreu um acidente de laboratório (EDWARDS, 1865). Na década de 50 houve um grande derramamento de $\mathrm{Hg}$ na Baía de Minamata (Japão) expondo a população a esse metal durante anos. Esse acontecimento chamou a atenção dos riscos à saúde pela exposição do $\mathrm{Hg}$, já que houve relato de vários casos de lesão cerebral profunda em recém-nascidos de mães que ingeriram peixes contaminados durante a gestação. Outro caso relatado na literatura ocorreu na zona rural do Iraque em 1970, onde agricultores e seus familiares ingeriram pão feito a partir de semente de trigo contaminado com o metal (CLARKSON, 2002; GOLDMAN; SHANNOM, 2001). Após esse ocorrido, exames histológicos de dois 
cérebros infantis revelaram que, uma das principais alterações patológicas foi, a ruptura da extensa citoarquitetura do cérebro. $\mathrm{O}$ exame microscópico revelou numerosos neurônios ectópicos localizados na substância cerebral branca e agrupamentos irregulares de neurônios corticais em muitas regiões, indicando uma perturbação aparente na migração de neurônios cerebrocorticais (CHOI et al., 1978.; CHOI, 1989). Incidentes semelhantes ocorreram na Guatemala, Paquistão e Gana (CLARKSON, 2002). Outros estudos demonstraram observações semelhantes com animais (KAKITA et al., 2001, KAKITA et al., 2002).

Diversas pesquisas sobre a exposição prénatal a $\mathrm{MeHg}$, em várias espécies de animais de laboratório e também em humanos, confirmam uma maior vulnerabilidade do cérebro fetal a esse metal (CASTOLDI et al., 2008; DREIEM et al., 2009; SAKAMOTO et al., 2013). De fato, estudos em animais focados nos efeitos do $\mathrm{MeHg}$ no cérebro durante o período de crescimento correspondente ao período final de gestação em humanos teve como resultado o desenvolvimento de um modelo experimental em ratos demonstrando uma degeneração neuronal no córtex cerebral e astrocitose (SAKAMOTO et al., 1993; WAKABAYASHI et al., 1995).

$\mathrm{O} \mathrm{MeHg}$ pode causar distúrbios persistentes no comportamento motor e alterações neuropatológicas gerais, incluindo morte de células neuronais e inflamação do nervo posterior em ratos. Atualmente, a exposição a baixos níveis de $\mathrm{MeHg}$ é comum entre a maioria das populações humanas, através do consumo de produtos do mar e peixes (MAHAFFEY et al., 2011).

\section{Absorção, Metabolismo e Excreção}

Compostos de $\mathrm{Hg}$ orgânico são solúveis em lipídeos e 95\% do $\mathrm{MeHg}$ ingerido a partir do consumo de peixes contaminados, é absorvido no trato gastrointestinal, embora o local exato de absorção não seja conhecido (NIELSEN; ANDERSEN, 1992). Uma vez no organismo, ele rapidamente converte-se em um complexo protéico, mantendo grande mobilidade através dos tecidos animais, o que também é facilitado pela sua lipossolubilidade (CERNICHIARI et al., 1995). O MeHg pode ser absorvido pela placenta e armazenado no cérebro fetal em concentrações que excedem os níveis sanguíneos maternos (níveis no cérebro fetal são cerca de 5-7 vezes maior que os do sangue materno) (PATRICK, 2002). O MeHg também pode atravessar facilmente a barreira hemato-encefálica (BHE), além de aparecer no leite materno e concentrarse nos rins e SNC (GRANDJEAN et al., 2010; SHANKER et al. 2005; YIN et al., 2007).

A meia-vida do $\mathrm{MeHg}$ varia de um tecido para o outro, mas geralmente é 45-70 dias (CLARKSON, 2002). O processo de distribuição do $\mathrm{MeHg}$ aos tecidos leva cerca de 30 horas. Aproximadamente $5 \%$ é encontrado na circulação sanguínea, 10\% no cérebro e $90 \%$ é excretado através das fezes. O $\mathrm{MeHg}$ está presente no corpo como um complexo solúvel em água, ligado principalmente com os grupamentos tióis do átomo de enxofre, e atravessa a BHE por complexação com a L-cisteína formando uma molécula semelhante a metionina (CLARKSON, 2002). O MeHg é liberado pelas células do organismo complexado com a glutationa reduzida, sendo degradado no ducto biliar em forma de complexos com uma L-cisteína. (BALLATORI; CLARKSON, 1985; OSAWA; MAGOS, 1974; PATRICK, 2002).

Somente $10 \%$ do $\mathrm{MeHg}$ é eliminado através dos rins e o restante retornam através da circulação entero-hepática ou é desmetilado pela microflora no intestino e sistema imunológico, sendo que a maior parte do $\mathrm{MeHg}$ eliminado encontra-se na forma inorgânica (CLARKSON, 2002). A conversão do $\mathrm{MeHg}$ em mercúrio inorgânico ocorre nas células fagocitárias do fígado ou nas células astrogliais do cérebro (CLARKSON, 2002). O mercúrio inorgânico tem sido encontrado como o principal 
forma no tecido cerebral em humanos fatalmente expostos ao MeHg (BALLATORI; CLARKSON, 1985; OSAWA; MAGOS, 1974; PATRICK, 2002).

\section{Toxicidade}

A exposição aguda ao $\mathrm{MeHg}$ provoca incoordenação motora, parestesia, ataxia, surdez, tremor muscular, constrição do campo visual,coma e até morte (AMIN-ZAKI et al., 1974; EKINO et al., 2007; GODMAN; SHANNON, 2001; MURATA et al., 2007).

A intoxicação crônica por $\mathrm{MeHg}$ provoca distúrbio da sensibilidade nas extremidades, disartria, surdez, constrição do campo visual, fraqueza muscular, irritabilidade, falta de memória, ansiedade, depressão, distúrbios de coordenação e equilíbrio e sinais motores que simula esclerose lateral amiotrófica e também parece aumentar o risco para doença cardiovascular (CHANG et al., 2008; CHUU; LIU; LIN-SHIAU, 2007; OZUAH, 2000).

$\mathrm{O} \mathrm{MeHg}$ apresenta diferenças na toxicocinética e toxicodinâmica no cérebro em desenvolvimento, visto que acumula-se mais no cérebro fetal que no materno. No cérebro em desenvolvimento, o $\mathrm{MeHg}$ é tóxico para o córtex cerebral e o cerebelo, devido a fatores intrínsecos do cérebro, causando destruição de neurônios e células gliais e exibe diferentes efeitos em relação ao SNC maduro (DREIEM; GERTZ; SEEGAL, 2005). No cérebro adulto, a intoxicação por $\mathrm{MeHg}$ danifica as chamadas zonas primárias do córtex cerebral, afeta o campo visual, vias auditivas, sensoriais somáticas e córtex motor. Além disso, a exposição ao $\mathrm{MeHg}$ prejudica o hipocampo e o cerebelo, causando uma intensa perda de neurônios nessas regiões do cérebro (DO NASCIMENTO et al., 2008; STEUERWALD et al., 2000).

Vários mecanismos têm sido propostos para explicar os efeitos neurotóxicos do $\mathrm{MeHg}$. Entre eles incluem-se o dano mitocondrial, indução de estresse oxidativo, interrupção da homeostase do cálcio intracelular, interrupção da função neurotransmissora, inibição dos receptores do ácido gama-aminobutírico (GABA), inibição da captação de glutamato e apoptose/necrose (ASCHNER et al., 2007; BELLÉ et al., 2009; DO NASCIMENTO et al., 2008; FERRARO et al., 2009; MORETTO et al., 2005; SHANKER et al., 2005; WAKABAYASHI et al., 1995).

A neurotoxicidade do $\mathrm{MeHg}$ vem sendo bastante explorada por diversos grupos de pesquisa. De fato, em ratos de 7 e 14 dias, o $\mathrm{MeHg}$ provoca uma perda no potencial de membrana mitocondrial em sinaptossomas, alteração nos níveis de dopamina e de seus metabólitos e aumento nas espécies reativas ao oxigênio (EROS), diminuindo assim os transportadores de dopamina, demonstrando que, de fato, o $\mathrm{MeHg}$ afeta o sistema dopaminérgico (DREIEM et al., 2009). Também em ratos com 7 dias, o $\mathrm{MeHg}$ provocou um aumento nas proteínas $\mathrm{BAX}$, citocromo $\mathrm{C}$ e posteriores aumentos na caspase 8 e 9, mesmo com baixas exposições ao $\mathrm{MeHg}$ in vivo. Já in vitro, a neurotoxicidade do $\mathrm{MeHg}$ é dependente da ativação da caspase 3 (SOKOLOWSKI et al., 2011).

No entanto, a exposição do cérebro a baixas concentrações de $\mathrm{MeHg}$ ainda tem sido escassamente explorada. Em um estudo utilizando ratas grávidas expostas a diferentes concentrações de $\mathrm{MeHg}$, observou-se uma diminuição da expressao de Rac 1, Cdc 42, RhoA e um posicionamento neuronal defeituoso podendo ser associada com a via de sinal Rho GTPase (GUO et al., 2013).

Em outro experimento, com ratas em período perinatal que foram expostas a baixas concentrações de $\mathrm{MeHg}$ observou-se um déficit na coordenação motora. Porém, apesar de não ter sido observado morte celular e inflamação do nervo, uma proteína que marca a formação sináptica, SPP (sinaptofisina), mostrou-se diminuída em grânulo cerebelar, demonstrando alterações nos sinaptossomas 
(FUJIMURA; CHENGB; WENCHZHAO, 2012).

Alguns modelos in vitro vêm sendo desenvolvidos para elucidar possíveis mecanismos envolvidos na neurotoxicidade do $\mathrm{MeHg}$. Entre os mecanismos envolvidos na neurotoxicidade do $\mathrm{MeHg}$ estão a depleção de glutationa (GSH) e o aumento da produção de espécies reativas ao oxigênio (EROS) (KAUR; ASCHNER; SYVERSEN, 2006; SARAFIAN; VERITY, 1991; SARAFIAN et al., 1994). Também foi observado a redução da expressão de ciclina $\mathrm{E}$ em exposição crônica e subtóxica ao $\mathrm{MeHg}$ sobre células pregenitoras neuronais (XU et al., 2010).

Outro aspecto de grande significância na toxicidade do $\mathrm{MeHg}$ refere-se aos efeitos de sua exposição no fígado. Estudos têm demonstrado que a exposição a diferentes metais pesados provocam danos ao tecido hepático sendo o fígado um órgão alvo importante para a toxicidade do $\mathrm{MeHg}$ (BORGES et al., 2008; MISHRA; FLORA, 2008; BRAGADIN et al., 2002).

$\mathrm{O} \mathrm{MeHg}$ possui alta afinidade com os tióis endógenos, resultando na depleção da GSH, um importante antioxidante intracelular. Em células hepáticas, o MeHg forma complexos solúveis com a cisteína e a glutationa, que são excretados na bile e reabsorvidos no trato gastrointestinal, iniciando um processo de peroxidação lipídica, o qual pode produzir alteração nas membranas celulares (ASHOUR; ABDEL-RAHMAN; KHODAIR, 1993; LIN; HUANG; HUANG, 1996). Além disso, o MeHg induz a abertura do poro de transição da permeabilidade nas mitocôndrias de fígado de ratos e esse fenômeno representa um importante mecanismo molecular responsável pela morte celular (BRAGADIN et al., 2002).

Poucas evidências foram estabelecidas a respeito dos efeitos da exposição a baixos níveis de $\mathrm{MeHg}$ na função renal em humanos, embora alguns estudos em animais demonstram que o $\mathrm{MeHg}$ induz toxicidade renal (NATIONAL RESEARCH COUNCIL, 2000; UNITED STATES
OF AMERICA, 1999). A exposição de ratos e camundongos ao $\mathrm{MeHg}$ em longo prazo provocou nefropatia crônica, degeneração epitelial dos túbulos proximais e fibrose intersticial (MITSUMORI et al., 1990). Também, outros estudos demonstram que quantidades significativas de $\mathrm{Hg}$ se acumulam nos rins após a exposição de formas orgânicas do mercúrio (ABDALLA et al., 2012; BRIDGES; ZALUPES, 2005; MAGOS et al., 1985; MCNEIL; BHATNAGAR; TURNER, 1988; ZALUPES et al., 1992) sugerindo que o mercúrio orgânico é oxidado a mercúrio inorgânico, antes e/ou após a sua entrada nas células epiteliais dos túbulos renais. No entanto, o nível de acumulação é muito menor do que a que ocorre após a exposição a formas inorgânicas ou elementares do mercúrio.

Estudos recentes em nosso laboratório, demonstraram que ratos jovens que receberam baixas doses de $\mathrm{MeHg}$ apresentaram um aumento da N-acetil- $\beta$-D-glucosaminidase (NAG), uma hidrolase lisossomal normalmente excretada em baixas quantidades na urina, que é considerada um indicador de nefrotoxicidade (ABDALLA et al., 2012; BAZZI et al., 2002; MANDI'C et al., 2005).

\section{Potenciais Agentes Protetores Experimentais}

Recentes estudos demonstram os efeitos de algumas substâncias com grande potencial contra a toxicidade do $\mathrm{MeHg}$. A vitamina $\mathrm{K}$ atua protegendo células neuronais, possivelmente por impedir a degradação de glutationa (GSH) intracelular, tendo sua capacidade de proteção contra os efeitos tóxicos no $\mathrm{MeHg}$ testada em uma cultura de células de cerebelo (SAKAUE et al., 2011).

O Selênio (Se) já é uma substância bem conhecida por sua atividade de proteção contra o $\mathrm{MeHg}$. Recentemente, foi desenvolvido um modelo animal pós-natal, onde o Se protegeu o córtex cerebral contra a degeneração neuronal e astrocitose provocadas pelo MeHg (SAKAMOTO et al., 2013).

Além disso, a utilização de plantas com poder 
antioxidante também vem sendo testada. Um exemplo interessante foi o demonstrado com uma exposição ao $\mathrm{MeHg}$ em ratas grávidas até o desmame dos filhotes associada com o uso da planta Rhododendron tomentosum ssp. subarcticum. Os resultados demonstraram um aumento no sistema antioxidante dos animais e dos níveis de N-metil-Daspartato (NMDA-R) (BLACK et al., 2011).

Também, o Allium sativium apresentou efeito protetor exposição a baixas doses de $\mathrm{MeHg}$ em hipocampo e córtex de ratos de 7 e 60 dias, in vitro (BELLÉ et al., 2009). O extrato alcoólico do bulbo de $A$. sativum foi capaz de reduzira inibição da adenosina deaminase(ADA) provocada pelo $\mathrm{MeHg}$. Os compostos sulfurosos presentes no extrato podem ter afetado a atividade da ADA, enzima chave no metabolismo adenosinergico do SNC (BELLÉ et al., 2009).

O Syzygium cumini (L.) Skeels é uma planta medicinal com uma grande quantidade de compostos fenólicos e tem sido estudada por ter demonstrado importantes atividades antioxidantes e anti-inflamatórias. $\mathrm{O}$ extrato aquoso dessa planta foi testado contra a toxicidade do $\mathrm{MeHg}$ em ratos jovens. A administração do extrato nos animais reverteu os efeitos tóxicos do $\mathrm{MeHg}$ em estruturas como cérebro, fígado e rins (ABDALLA et al., 2011).

Possíveis mecanismos envolvidos na mitigação dos efeitos do $\mathrm{MeHg}$ por fitoquímicos podem incluir a redução das EROS, a ativação dos sistemas antioxidantes enzimáticos, a restauração do potencial da membrana mitocondrial, a modulação de vias de sinalização celular (CAMPOSESPARZA; SANCHEZ-GOMEZ; MATUTE, 2009). Fitoquímicos também pode afetar a biodisponibilidade e farmacocinética do $\mathrm{MeHg}$ (LAIRD et al., 2009; PASSOS et al., 2007).

\section{Considerações Finais}

Os conceitos aqui expostos contribuem para a compreensão sobre a toxicidade do $\mathrm{MeHg}$, um poluente ambiental de grande risco à saúde humana. É notório considerar que os efeitos biológicos deste metal abrangem alterações citológicas, reprodutivas e, especialmente neurológicas. Notadamente, durante o período de desenvolvimento estes efeitos são relevantes, pois demonstram deficiências neurológicas e motoras significativas. Deste modo, o desenvolvimento de estudos experimentais têm sido fundamental para conhecer os efeitos tóxicos do $\mathrm{MeHg}$, e também para as investigações de prováveis alvos moleculares que possam minimizar os efeitos deste metal pesado.

\section{Agradecimentos}

Os autores agradecem ao Conselho Nacional de Desenvolvimento Científico e Tecnológico (CNPq), a Coordenação de Aperfeiçoamento de Pessoal de Nível Superior (CAPES) e a Universidade Federal de Santa Maria (UFSM) pelo suporte para a realização desse estudo.

\section{Referências}

ABDALLA, F. H.; BELLÉ, L. P.; BITENCOURT, P. E. R.; DE BONA, K. S.; ZANETTE, R. A.; BOLIGON, A. A.; ATHAYDE, M. L.; PIGATTO, A. S.; MORETTO, M. B. Protective effects of Syzygium cumini seed extract against methylmercury-induced sistemic toxicity in neonatal rats. Biometals, London, v. 24, p. 349-356, 2011.

ABDALLA, F. H.; BELLÉ, L. P.; BITENCOURT, P. E. R.; SILVA, J. E. P.; ROMAN, S.; ROSA, C.; SCHETINGER, M. R.; MORETTO, M. B. Methylmercury-induced changes in target organs of suckling rat pups. Experimental and Toxicologic 
Pathology, New York, v. 64, p. 605-609, 2012.

AMIN-ZAKI, L.; ELHASSANI, S.; MAJEED, M. A.; CLARKSON, T. W.; DOHERTY, R. A.; GREENWOOD, M. Intra-uterine methylmercury poisoning in Iraq. Pediatrics, New York, v. 54, p. 587-595, 1974.

ASCHNER, M.; SYVERSEN, T.; SOUZA, D. O.; ROCHA, J. B.; FARINA, M. Involvement of glutamate and reactive oxygen species in methylmercury neurotoxicity. Brazilian Journal of Medical and Biological Research, Ribeirão Preto, v. 40, p. 285-291, 2007.

ASHOUR, H.; ABDEL-RAHMAN, M.; KHODAIR, A. The mechanism of methyl mercury toxicity in isolated rat hepatocytes. Toxicology Letters, Amsterdam, v. 69, n. 1, p. 87-96, 1993.

AUCOTT, M.; MCLINDEN, M.; WINKA, M. Release of mercury from broken fluorescent bulbs. Journal of the Air \& Wast Management Association, Pittsburgh, v. 53, n. 2, p. 143-151, 2003.

BALLATORI, N.; CLARKSON, T. W. Sulfobromophthalein inhibition of glutathione and methylmercury secretion into bile. American Journal Physiology, Washington, v. 248, p. 238245, 1985.

BAZZI, C.; PETRINI, C.; RIZZA, V.; ARRIGO, G.; NAPODANO, P.; PAPARELLA, M.; D'AMICO, G. Urinary N-acetyl- $\beta$-D-glucosaminidase excretion is a marker of tubular cell disfunction and a predictoro outcome in primary glomerulunephritis. Nephrology Dialysis Transplantation, v. 17, p. 1890-96, 2000.

BELLÉ, L. P.; DE BONA, K. S.; ABDALLA, F. H.; PIMENTEL, V. C.; PIGATTO, A. S.; MORETTO, M. B. Comparative Evaluation of Adenosine Deaminase Activity in Cerebral Cortex and Hippocampus of Young and Adult Rats: Effect of Garlic Extract (Allium sativum L.) on Their Susceptibility to Heavy Metal Exposure. Basic \& Clinical Pharmacology \& Toxicology, Copenhagen, v. 104, n. 5, p. 408-413, 2009.
BLACK, P.; NIU, L.; SACHDEVA, M.; LEAN, D.; POON, R.; BOWERS, W. J.; CHAN, H. M.; ARNASON, J. T.; PELLETIER, G. Modulation of the effects of methylmercury on rat neurodevelopment by co-exposure with Labrador Tea (Rhododendron tomentosum ssp. subarcticum). Food and Chemical Toxicology, Oxford, v. 49, p. 2336-2342, 2011.

BORGES, L. P.; BRANDÃO, R.; GODOI, B.; NOGUEIRA, C. W.; ZENI, G. Oral administration of diphenyl diselenide protects against cadmiuminduced liver damage in rats. Chemical Biological Interactions, Amsterdam, v. 171, n. 1, p. 15-25, 2008.

BRAGADIN, M.; MARTON, D.; MANENTE, S.; GRASSO, M.; TONINELLO, A. Methylmercury induces the opening of the permeability transition pore in rat liver mitochondria. Journal of Inorganic Biochemistry, New York, v. 89, n. 1/2, p. 159-62, 2002.

BRIDGES, C. C.; ZALUPS, R. K. Molecular and ionic mimicry and the transport of toxic metals. Toxicology Applied Pharmacology, New York, v. 204, n. 3, p. 274-308, 2005.

CAMPOS-ESPARZA, M. R.; SANCHEZGOMEZ, M. V.; MATUTE, C. Molecular mechanisms of neuroprotection by two natural antioxidant polyphenols. Cell Calcium, Edinburgh, v. 45, n. 4, p. 358-368, 2009.

CANELA, M. C. Determinação de Mercúrio a nivel traço: aplicação em amostras de interesse ambiental. 1995. 113 f. Dissertação (Mestrado em Química Analítica) - Universidade Estadual de Campinas, Campinas, 1995.

CARVALHO, C. M. L.; MATOS, A. I. N. M.; MATEUS, M. L.; SANTOS, A. P. M.; BATOREU, M. C. C. High-fish consumption and risk prevention: assessment of exposure to methylmercury in Portugal. Journal Toxicology Environmental Health, Washington, v. 71, n. 18, p. 1279-1288, 2008.

CASTOLDI, A. F.; ONISHCHENKO, 
N.; JOHANSSON, C. T.; RODA, C. E.; VAHTER, M.; CECCATELLI, S.; MANZO, L. Neurodevelopmental toxicity of methylmercury: laboratory animal data and their contribution to human risk assessment. Regulatory Toxicology Pharmacology, New York, v. 51, n. 2, p. 215-229, 2008.

CERNICHIARI, E.; BREWER, R.; MYERS, G. J.; MARSH, D. O.; LAPHAM, L. W.; COX, C.; SHAMLAYE, C. F.; BERLIN, M.; DAVIDSON, P. W.; CLARKSON, T. W. Monitoring methylmercury during pregnancy: maternal hair predicts fetal brain exposure. Neurotoxicology, Park Forest South, v. 16, n. 4, p.705-710, 1995.

CHANG, J. W.; PAI, M. C.; CHEN, H. L.; GUO, H. R.; SU, H. J.; LEE, C. C. Cognitive function and blood methylmercury in adults living near a deserted chloralkali factory. Environmental Research, San Diego, v. 108, p. 334-339, 2008.

CHOI, B. H. The effects of methylmercury on the developing brain. Progress in Neurobiology, New York, v. 32, n. 6, p. 447-470, 1989.

CHOI, B. H.; LAPHAM, L. W.; AMIN-ZAKI, L.; SALEEM, T. Abnormal neuronal migration, deranged cerebral cortical organization, and diffuse white matter astrocytosis of human fetal brain: a major effect of methylmercury poisoning in utero. Journal of Neuropathology Experimental Neurologic, Lawrence, v. 37, n. 6, p. 719-733, 1978.

CHUU, Y. Y.; LIU, S. H.; LIN-SHIAU, S. Differential neurotoxic effects of methylmercury and mercuric sulfide in rats. Toxicology Letters, Amsterdam, v. 169, p. 109-120, 2007.

CLARKSON, T. W. The three modern faces of mercury. Environmental Health Perspective, Research Triangle Park, v. 110, p. 11-23, 2002.

DO NASCIMENTO, J. L.; OLIVEIRA, K. R.; CRESPO-LOPEZ, M. E.; MACCHI, B. M.; MAUÉS, L.A.; PINHEIRO, M. D.A. C.; SILVEIRA, L. C.; HERCULANO, A. M. Methylmercury neurotoxicity \& antioxidant defenses. Indian
Journal Medical Research, New Delhi, v. 128, n. 4, p. 373-82, 2008.

DREIEM, A.; GERTZ, C. C.; SEEGAL, R. F. The effects of methylmercury on mitochondrial function and reactive oxygen species formation in rat striatal synaptosomes are age-dependent. Toxicology Science, Orlando, v. 87, n. 1, p. 156-62, 2005.

DREIEM, A.; SHAN, M.; OKONIEWSKI, R. J.; MORRISSEY, S. S.; SEEGAL, R. F. Methylmercury inhibits dopaminergic function in rat pup synaptosomes in an age-dependent manner. Neurotoxicology and Teratology, Elmsford, v. 31, n. 5, p. 312-317, 2009.

EDWARDS, G. N. Two cases of poisoning by mercuric methide. Saint Bartholomew's Hospital, London, v. 1, p. 1-10, 1865.

EKINO, S.; NINOMIYA, T.; IMAMURA, K.; SUSA, M. Methylmercury causes diffuse damage to the somatosensory cortex: how to diagnose Minamata disease. Seishin Shinkeigaku Zasshi, Tokyo, v. 109, n. 5, p. 420-437, 2007.

FERRARO, L.; TOMASINI, M. C.; TANGANELLI, S.; MAZZA, R.; COLUCCIA, A.; CARRATU, M. R.; GAETANI, S.; CUOMO, V.; ANTONELLI, T. Developmental exposure to methylmercury elicits early cell death in the cerebral cortex and long-term memory deficits in the rat. International Journal Development Neuroscience, Oxford, v. 27, n. 2, p. 165-174, 2009.

FUJIMURA, M.; CHENGB, J.; WENCHZHAO, W. Perinatal exposure to low-dose methylmercury induces dysfunction of motor coordination with decreases in synaptophysin expression in the cerebellar granule cells of rats. Brain Reseach, Amsterdam, v. 1464, p. 1-7, 2012.

GOLDMAN, L. R.; SHANNON, M. W. American academy of pediatrics: committee on environmental health technical report: mercury in environment: implications for pediatricians. Pediatrics, New York, v. 108, p. 197-205, 2001. 
GRANDJEAN, P.; SATOH, H.; MURATA, K.; ETO, K. Adverse effects Oof methylmercury: environmental health research implications. Environmental Health Perspectives, Research Triangle Park, v. 118, n. 8, p. 1137-1145, 2010.

GRESSESN, P.; MESPLES, B.; SAHIR, N.; MARRET, S.; SOLA, A. Environmental factores and disturbances of brian development. Seminars in neonatology, London, v. 6, n. 2, p. 185-194, 2001.

GUO, B. Q.; YAN, C. H.; CAI, S. Z.; YUAN, X. B.; SHEN, X. M. Low level prenatal exposure to methylmercury disrupts neuronal migration in the developing rat cerebral cortex. Toxicology, Amsterdam, v. 304, p. 57-68, 2013.

HAYKAL-COATES, N.; SHAFER, T. J.; MUNDY, W. R.; BARONE, S. Effects os gestacional methylmercury exposure on immunoreactivity of specific isoforms of PKC and enzymes activity during pos-natal development of the rat brain. Developmental brain research, Amsterdam, v. 109, p. 33-49, 1998.

HORVAT, M. Mercury analyses and speciation. In: BAEYENS, P. M.; EBINGHAUS, W. R.; VASILIER, O. Global and regional mercury cycles: sources, fluxes and mass balances. Cordrecht: Kluwer Academic, 1996. v. 21. p. 1-31.

JARDIM, W. F. Contaminação por mercúrio: Fatos e Fantasias. Ciência Hoje, São Paulo, v. 41, p. 78-79, 1988.

JUAREZ, B. I.; MARTINEZ, M. L.; MONTANTE, M.; DUFOUR, L.; GARCIA, E.; JIMENEZ-CAPDEVILLE, M. E. Methylmercury increase glutamate extracellular levels in frontal cortex of awake rats. Neurotoxicology and teratology, Oxford, v. 5516, p. 1-5, 2002.

KAKITA, A.; INENAGA, C.; SAKAMOTO, M.; TAKAHASHI, H. Neuronal migration disturbance and consequent cytoarchitecture in the cerebral cortex following transplacental administration of methylmercury. Acta Neuropathologica, Berlin, v. 104, n. 4, p. 409-417, 2002.
KAKITA, A.; WAKABAYASHI, K.; SU, M.; PIAO, Y. S.; TAKAHASHI, H. Experimentally induced leptomeningeal glioneuronal heterotopia and underlying cortical dysplasia of the lateral limbic area in rats treated transplacentally with methylmercury. Journal Neuropathology Experimental Neurology, Lawrence, v. 60, n. 8, p. 768-777, 2001.

KAKITA, A.; WAKABAYASHI, K.; SU, M.; YONEOKA, Y.; SAKAMOTO, M.; IKUTA, F.; TAKAHASHI, H. Intrauterine methylmercury intoxication. Consequence of the inher brain lesions and cognitive dysfunction in maturity. Brain research, Amsterdam, v. 887, p. 322-30, 2000.

KAUR, P.; ASCHNER, M.; SYVERSEN, T. Glutathione modulation influences methyl mercury induced neurotoxicity in primary cell cultures of neurons and astrocyte. Neurotoxicology, Little Rock, v. 27, p. 492-500, 2006.

LAIRD, B. D.; SHADE, C.; GANTNER, N.; CHAN, H. M.; SICILIANO, S. D. Bioaccessibility of mercury from traditional northern country foods measured using an in vitro gastrointestinal model is independent of mercury concentration. Science Total Environmental, Amsterdam, v. 407, n. 23, p. 6003-6008, 2009.

LIN, T. H.; HUANG, Y. L.; HUANG, S. F. Lipid peroxidation in liver of rats administrated with methyl mercuric chloride. Biological Trace Element Research, London, v. 54, n. 1, p. 33-41, 1996.

MAGOS, L.; BROWN, A.W.; SPARROW, S.; BAILEY, E.; SNOWDEN, R.T.; SKIPP, W.R. The comparative toxicology of ethyl- and methylmercury. Archives Toxicology, Berlin, v. 57, n. 4, p. 260-267, 1985.

MAHAFFEY, K. R.; SUNDERLAND, E. M.; CHAN, H. M.; CHOI, A. L.; GRANDJEAN, P.; MARIEN, K.; OKEN, E.; SAKAMOTO, M.; SCHOENY, R.; WEIHE, P.; YAN, C. H.; YASUTAKE, A. Balancing the benefits of $n-3$ polyunsaturated fatty acids and the risks of 
methylmercury exposure from fish consumption. Nutrition Reviews, New York, v. 69, p. 493-508, 2011.

MANDI'C, L. M.; ACIMOVIC, J. M.; JOVANOVIC, V. B. The posibility of determining $\mathrm{N}$-acetyl-beta-D-glucosaminidase isoenzymes under alkaline conditions. Clinical Biochemistry, v. 38, p. 384-389, 2005.

MCNEIL, S. I.; BHATNAGAR, M. K.; TURNER, C. J. Combined toxicity of ethanol and methylmercury in rat. Toxicology, Amsterdam, v. 53, n. 2/3, p. 345-363, 1988.

MICARONI, R. C. C. M.; BUENO, M. I. M.; JARDIM, W. F. Compostos de mercúrio: revisão de métodos de determinação, tratamento e descarte. Química Nova, São Paulo, v. 23, p. 487-495, 2000.

MISHRA, D.; FLORA, S. J. Quercetin administration during chelation therapy protects arsenic-induced oxidative stress in mice. Biological Trace Elementary Research, London, v. 122, n. 2, p. 137-147, 2008.

MITSUMORI, K.; HIRANO, M.; UEDA, H.; MAITA, K.; SHIRASU, Y. Chronic toxicity and carcinogenicity of methylmercury chloride in B6C3F1 mice. Fundamental Applied Toxicology, Akron, v. 14, n. 1, p. 179-190, 1990.

MORETTO, M. B.; FUNCHAL, C.; SANTOS, A. Q.; GOTTFRIED, C.; BOFF, B.; ZENI, G.; PUREUR, R. P.; SOUZA, D. O.; WOFCHUK, S.; ROCHA, J. B. Ebselen protects glutamate uptake inhibition caused by methyl mercury but does not by $\mathrm{Hg} 2+$. Toxicology, Amsterdam, v. 214, n. 1/2, p. 57-66, 2005.

MURATA, K.; DAKEISHI, M.; SHIMADA, M.; SATOH, H. Assessment of intrauterine methylmercury exposure affecting child development: messages from the newborn. Tohoku Journal of Experimental Medicine, Sendai, v. 213, n. 3, p. 187-202, 2007.

\section{NATIONAL RESEARCH COUNCIL.}

Toxicological effects of methylmercury. Washington: National Academy Press, 2000.

NIELSEN, J. B.; ANDERSEN, O. The toxicokinetics of mercury in mice offspring after maternal exposure to methylmercury-effect of selenomethionine. Toxicology, Amsterdam, v. 74, n. 2/3, p. 233-241, 1992.

OSAWA, M.; MAGOS, L. The chemical form of the methylmercury complex in the bile of the rat. Biochemical Pharmacology, New York, v. 23, p. 1903-1905, 1974.

OZUAH, P. O. Mercury poisoning. Currents: Current Problems in Pediatrics, St. Louis, v. 3, n. 3, p. 91-99, 2000.

PASSOS, C. J.; MERGLER, D.; FILLION, M.; LEMIRE, M.; MERTENS, F.; GUIMARAES, J. R.; PHILIBERT, A. Epidemiologic confirmation that fruit consumption influences mercury exposure in riparian communities in the Brazilian Amazon. Environmental Research, San Diego, v. 105, p. 183-193, 2007.

PATRICK, L. N. D. Mercury toxicity and antioxidants: part I: role of glutathione and alphaLipoic acid in the treatment of mercury toxicity. Alternative Medicine Review, Sandpoint, v. 7, n. 6, p. 456-471, 2002.

SAKAMOTO, M.; NAKANO, A.; KAJIWARA, Y.; NARUSE, I.; FUJISAKI, T. Effects of methyl mercury in postnatal developing rats. Enviromental Research, San Diego, v. 61, n. 1, p. 43-50, 1993.

SAKAMOTO, M.; YASUTAKE, A.; KAKITA, A.; RYUFUKU, M.; CHAN, H.M.; YAMAMOTO, M.; OUMI, S.; KOBAYASHI, S.; WATANABE, C. Selenomethionine protects against neuronal degeneration by methylmercury in the developing rat cerebrum. Environmental Science \& Technology, v. 47, n. 6, p. 2862-2868, 2013.

SAKAUE, M.; MORI, N.; OKAZAKI, M.; KADOWAKI, E.; KANEKO, T.; HEMMI, N.; SEKIGUCHI, H.; MAKI, T.; OZAWA, A.; HARA, 
S.; ARISHIMA, K.; YAMAMOTO, M. Vitamin $\mathrm{K}$ Has the potential to protect Neurons from methylmercury-induced cell death in vitro. Journal of Neuroscience Research, New York, v. 89, p. 1052-1058, 2011.

SANFELIU, C.; SEBASTIAN, J.; KIM, S. U. Methylmercury neurotoxicity in culture of humans neurons astrocytes, neuroblastoma cells. Neutotoxicology, Park Forest South, v. 22, n. 3, p. 317-327, 2001

SARAFIAN, T.A.; VARTAVARIAN, L.; KANE, D. J.; BREDESEN, D. E.; VERITY, M. A. Bcl-2 expression decreases methylmercury-induced freeradical generation and cell killing in a neural cell line. Toxicology Letters, Amsterdam, v. 74, n. 2, p. 149-155, 1994.

SARAFIAN, T. A.; VERITY, M. A. Oxidative mechanisms underlying methylmercury neurotoxicity. International Journal of Development Neuroscience, Oxford, v. 9, n. 2, p. 147-153, 1991.

SHANKER, G.; MUTKUS, L. A.; WALKER, S. J.; ACHNER. M. Methylmercury enhances arachidonic acid release and cytosolic phospholipase A2 expression in primary cultures of neonatal astrocytes. Molecular Brain Research, Amsterdam, v. 106, p. 1-11, 2002.

SHANKER, G.; SYVERSEN, T.; ASCHNER, J. L.; ASCHNER, M. Modulatory effect of glutathione status and antioxidants on methylmercury-induced free radical formation in primary cultures of cerebral astrocytes. Molecular Brain Research, Amsterdam, v. 137, p. 11-22, 2005.

SOKOLOWSKI, K.; MOREL, A. F.; ZHOU, X.; BLOOM, E. D. Methylmercury (MeHg) elicits mitochondrial-dependent apoptosis in developing hippocampus and acts at low exposures. NeuroToxicology, Little Rock, v. 32, p. 535-544, 2011.

STEUERWALD, U.; WEIHE, P.; JORGENSEN, P.J.; BJERVE, K.; BROCK, J.; HEINZOW, B.; BUDTZ-JØRGENSEN, E.; GRANDJEAN, P.
Maternal seafood diet, methylmercury exposure and neonatal neurologic function. Journal Pediatrics, St. Louis, v. 136, n. 5, p. 599-605, 2000.

TROMBETTA, L. D.; KROMIDAS, L. A scanning electron-microscopic study of the effects of methylmercury on the neuronal cytoskeleton. Toxicology Letters, Amsterdam, v. 60, p. 329-341, 1992.

UNITED NATIONS ENVIRONMENT PROGRAMME. Global Mercury Assessment. Geneva, 2002.

UNITED STATES OF AMERICA. Department of Health and Human Services. Toxicological profile for mercury. Atlanta, 1999. Disponível em: $<$ http:// www.atsdr.cdc.gov/toxprofiles/tp46.pdf $>$. Acesso em: 15 dez. 2012.

WAKABAYASHI, K.; KAKITA, A.; SAKAMOTO, M.; SU, M.; IWANAGA, K.; IKUTA, F. Variability of brain lesions in rats administered methylmercury at various postnatal development phases. Brain Research, Amsterdam, v. 705, p. 267-72, 1995.

XU, M.; YAN, C.; TIAN, Y.; YUAN, X.; SHEN, X. Effects of low level of methylmercury on proliferation of cortical progenitor cells. Brain Research, Amsterdam, v. 1359, p. 272-280, 2010.

YIN, Z.; MILATOVIC, D.; ASCHNER, J. L.; SYVERSEN, T.; ROCHA, J. B.; SOUZA, D. O.; SIDORYK, M.; ALBRECHT, J.; ASCHNER, M. Methylmercury induces oxidative injury, alterations in permeability and glutamine transport in cultured astrocytes. Brain Research, Amsterdam, v. 1131, p. 1-10, 2007.

ZALUPES, R. K.; BARFUSS, D. W.; KOSTYNIAK, P. J. Altered intrarenal accumulation of mercury in uninephrectomized rats treated with methylmercury chloride. Toxicology Applied Pharmacology, v. 115, p. 174-82, 1992.

Recebido em: 29 de abril de 2011 Aceito em: 11 de julho de 2013 
